The University of Southern Mississippi

The Aquila Digital Community

Faculty Publications

8-2018

\title{
Myocardial Stunning-Induced Left Ventricular Dyssynchrony On Gated Single-Photon Emission Computed Tomography Myocardial Perfusion Imaging
}

\author{
Zhixin Jiang \\ The First Affiliated Hospital of Nanjing Medical University \\ Haipeng Tang \\ University of Southern Mississippi \\ Jianzhou Shi \\ The First Affiliated Hospital of Nanjing Medical University \\ Yanli Zhou \\ The First Affiliated Hospital of Nanjing Medical University \\ Cheng Wang \\ The Frist Affiliated Hospital of Nanjing Medical University
}

See next page for additional authors

Follow this and additional works at: https://aquila.usm.edu/fac_pubs

Part of the Analytical, Diagnostic and Therapeutic Techniques and Equipment Commons

\section{Recommended Citation}

Jiang, Z., Tang, H., Shi, J., Zhou, Y., Wang, C., Li, D., Shan, Q., Zhou, W. (2018). Myocardial Stunning-Induced Left Ventricular Dyssynchrony On Gated Single-Photon Emission Computed Tomography Myocardial Perfusion Imaging. Nuclear Medicine Communications, 39(8), 725-731.

Available at: https://aquila.usm.edu/fac_pubs/15486

This Article is brought to you for free and open access by The Aquila Digital Community. It has been accepted for inclusion in Faculty Publications by an authorized administrator of The Aquila Digital Community. For more information, please contact Joshua.Cromwell@usm.edu. 


\section{Authors}

Zhixin Jiang, Haipeng Tang, Jianzhou Shi, Yanli Zhou, Cheng Wang, Dianfu Li, Qijun Shan, and Weihua Zhou

This article is available at The Aquila Digital Community: https://aquila.usm.edu/fac_pubs/15486 


\section{Myocardial stunning induced left ventricular dyssynchrony on gated SPECT myocardial perfusion imaging}

Zhixin Jiang, MD a, Haipeng Tang, MS ${ }^{\text {b }}$, Jianzhou Shi, MS a, Yanli Zhou, MD a, Cheng Wang, MD a, Dianfu Li, MD a, Weihua Zhou, PhD b* ${ }^{*}$ Qijun Shan, MD a ${ }^{*}$

(CONTRIBUTION OF EACH AUTHOR: Zhixin Jiang - conception and design and analysis and interpretation of data; drafting of the manuscript. (2) Haipeng Tang, Jianzhou Shi, Yanli Zhou, Cheng Wang, Dianfu $\mathrm{Li}$ - active involvement in collecting data and performing experiments with subsequent participation in data analysis. (3) Qijun Shan - revising it critically for important intellectual content. (4) Weihua Zhou — final approval of the manuscript submitted)

a Department of Cardiology, The First Affiliated Hospital of Nanjing Medical University, China b School of Computing, University of Southern Mississippi, Long Beach, MS

\section{Running Title: Myocardial stunning induced left ventricular dyssynchrony}

* Corresponding authors:

Qijun Shan

Tel: +862568136407

E-mail address: gjshan@njmu.edu.cn

Mailing address: 300 Guangzhou Road, Nanjing 210029, China 
or

Weihua Zhou

Tel: +1 228-214-3265

E-mail address: weihua.zhou@usm.edu

Mailing address: 730 East Beach Blvd, Long Beach, MS, 39560, USA

DISCLOSURES The authors have no conflicts of interest to disclose.

Funding This research was supported in part by an AHA grant (17AIREA33700016, PI: Weihua Zhou, PhD) and a New Faculty startup grant from the University of Southern Mississippi (DE01791, PI: Weihua Zhou).

(words: main text 5007, abstract 240) 


\section{Abstract}

Objectives: Myocardial stunning provides additional non-perfusion markers of coronary artery disease (CAD), especially for severe multi-vessel CAD. The purpose of this study is to assess the influence of myocardial stunning to the changes of left ventricular mechanical dyssynchrony (LVMD) parameters between stress and rest gated single photon emission computed tomography (SPECT) myocardial perfusion imaging (MPI).

Methods: 113 consecutive patients (88 males and 25 females) who had undergone both stress and rest Tc-99m sestamibi gated SPECT MPI were retrospectively enrolled. Suspected or known CAD patients were included if they had exercise stress MPI and moderate to severe myocardial ischemia. Segmental scores were summed for the three main coronary arteries according to standard myocardial perfusion territories and then regional perfusion, wall motion and wall thickening scores were measured. Myocardial stunning was defined as both ischemia and wall dysfunction within the same coronary artery territory. Patients were divided into the stunning group $(\mathrm{n}=58)$ and non-stunning group $(\mathrm{n}=55)$.

Results: There was no significant difference of LVMD parameters between stress and rest in the non-stunning group. In the stunning group, phase standard deviation (PSD) and phase histogram bandwidth $(\mathrm{PHB})$ of contraction were significantly larger during stress than during rest $(15.05 \pm$ 10.70 vs. $13.23 \pm 9.01$ and $46.07 \pm 34.29$ vs. $41.02 \pm 32.16, p<0.05)$. PSD and PHB of relaxation were also significantly larger during stress than during rest $(21.21 \pm 13.91$ vs. $17.46 \pm 10.52$ and $59.03 \pm 37.82$ vs. $52.38 \pm 36.89, p<0.05)$.

Conclusions: Both systolic and diastolic LVMD parameters deteriorate with myocardial stunning. This kind of change may have incremental values to diagnose CAD.

【Keywords】 coronary artery disease; left ventricular dyssynchrony; myocardial perfusion 
imaging; myocardial stunning 


\section{Introduction}

Phase analysis on gated single photon emission computed tomography (SPECT) myocardial perfusion imaging (MPI) is well established to measure left ventricular mechanical dyssynchrony (LVMD)[1]. LVMD has been found to relate with stress-induced myocardial ischemia [2-5]. However, contradictory findings exist among these studies. Chen et al[3] demonstrated different LVMD changes in ischemic, infarcted and normal myocardium using gated TI-201 SPECT $\mathrm{MPI}$, which was acquired at $5-10$ minutes after the start of stress. They found that in the ischemia group, LVMD parameters were significantly larger during stress than during rest. However, Singh et al[4] reported a different result that early-stress LVMD parameters were lower as compared to that at rest using TI-201 gated MPI. When Tc-99m-sestamibi was used, even presence of large reversible perfusion defects (reversible perfusion defects involving $>10 \%$ of the LV myocardium) did not alter the indices of LVMD because MPI was acquired 45-60 minutes post stress[6]. Zhou et al[5] also reported no significant difference in LVMD parameters between rest and 60 minutes after stress on gated MPI using Tc-99m sestamibi. However, other researchers found the evidence of stress-induced LVMD with Tc-99m-sestamibi when acquisitions were performed earlier than an hour post stress [7-9].

Myocardial stunning is defined as myocardium with persistent contractile dysfunction despite the restoration of perfusion after a period of ischemia[10]. It lasts from minutes to days, which depends on the duration and severity of ischemia. LVMD in stress-induced ischemia is associated with stress-induced stunning, and different extent of ischemia burden may ascribe to the different results among these studies [2-9]. Direct analysis about the correlation between LVMD and myocardial stunning may eliminate the influence of ischemia burden and stress acquisition time. The aim of this study is to assess the influence of myocardial stunning to the

changes of LVMD parameters between stress and rest gated SPECT MPI in patients with 
suspected or known coronary artery disease (CAD).

\section{Materials and methods}

\section{Patient population}

A group of 1503 consecutive patients who had undergone both stress and rest Tc-99m sestamibi myocardial perfusion SPECT in our center between January 2014 and August 2016 were retrospectively enrolled. Patients were included if they had exercise stress SPECT MPI and moderate to severe myocardial ischemia. Patients with history of pacemaker implantation, known rhythm abnormality (left and right bundle branch block) and ejection fraction (EF) $<40 \%$ were excluded from the study. Patients who did not reach $a \geq 85 \%$ heart rate during exercise test were also excluded. Finally, data of 113 patients (88 males and 25 females, $61.6 \pm 5.65$ years old) with suspected or known CAD were processed. The retrospective study was approved by the Institutional Ethical Committee of the First Affiliated Hospital of Nanjing Medical University.

\section{Image acquisition and processing}

The image acquisition and processing protocol were the same as those reported by our center [11]. A two-day Tc-99m sestamibi protocol was used. Patients underwent a symptom limited treadmill test using standard Bruce protocol. Tc-99m sestamibi was injected when a $\geq 85 \%$ heart rate was achieved. Both stress and rest image acquisitions were started at 60 minutes after the administration of Tc-99m sestamibi. Gated stress/rest myocardial SPECT was acquired using the Philips CardioMD dual-head cameras with low energy high-resolution collimators. An ECG R-wave detector provided gating to acquire 8 frames per cardiac cycle. The R-R acceptance window for beat rejection was set to the average R-R duration $\pm 50 \%$. Images were acquired over a $180^{\circ}$ noncircular orbit from $45^{\circ}$ right anterior oblique to $45^{\circ}$ left posterior oblique, with 32 seconds per projection, $64 \times 64$ matrix and $140 \mathrm{keV} \pm 20 \%$ energy window for emission images. Tomographic 
reconstruction and oblique reorientation were done using Auto-SPECT-Plus on JetStream (Philips Medical Systems, Milpitas, CA). Planar images were reconstructed by filter back projection with a Butterworth filter (order $=5$ and cutoff frequency $=0.66$ ). No attenuation correction was applied.

\section{Data interpretation}

Gated SPECT data were processed using quantitative cardiac software packages and the LV myocardium was divided into 17 segments following the AHA/ACC/ASNC guidelines. Summed stress score (SSS), summed rest score (SRS) and summed difference score (SDS) were calculated using the QPS software package (Cedars Sinai, Los Angeles, CA, USA). Regional summed difference score (SDSr) was calculated for the three main coronary arteries, according to standard myocardial perfusion territories [12,13]. Stress and rest wall motion score (WMS) and wall thickening score (WTS) were analyzed using the QGS software package (Cedars Sinai, Los Angeles, CA, USA). Regional summed difference wall motion score (WMSr) and reginal summed difference wall thickening score (WTSr) were also calculated. Myocardial stunning was defined as both ischemia and wall dysfunction within the same coronary artery territory (SDSr $\geq 1$ and WMSr $+\mathrm{WTS} r \geq 1$ ). According to myocardial stunning and SRS, four subgroups were then generated: Group 1, myocardial ischemia without stunning (SRS $\leq 3,35$ patients); Group 2, myocardial ischemia with stunning (SRS $\leq 3,38$ patients); Group 3, myocardial ischemia and infarction

without stunning (SRS > 3, 20 patients) and Group 4, myocardial ischemia and infarction with stunning (SRS $>3,20$ patients).

End-diastolic volume (EDV), end-systolic volume (ESV) and EF were analyzed using QGS. Transient ischemia dilation (TID) was measured by QPS. LVMD parameters (characterized by phase standard deviation [PSD] and phase histogram bandwidth [PHB]) were measured with Emory Cardiac Toolbox (Atlanta, GA, USA).

\section{Coronary angiography}

35 of 113 patients underwent coronary angiography within 3 months after gated SPECT 
MPI. At least 2 orthogonal views were obtained and the projection showing the most severe stenosis was used for quantitative coronary measurements. Considering the mean proximal and distal reference diameters, the percentage lumen reduction was calculated offline by two experienced investigators. Multi-vessel CAD was defined as more than two main coronary arteries presented with stenosis $>70 \%$ and single-vessel CAD was defined as only one main coronary artery presented with stenosis $>70 \%$.

\section{Statistical analysis}

Continuous data were expressed as mean \pm SD and categorical data as number and percentage. Differences in baseline characteristics were analyzed by unpaired student tests (continuous data) or chi-square tests (dichotomous data). Non-parametric correlation (Spearman correlation) was used to study the relationship between SSS, LVMD, WMS and WTS. The paired t-test was used to compare the changes in PSD, PHB, EDV, ESV and EF from rest to stress in each group. The area under the receiver operating characteristic (ROC) curves was determined to assess the performance of myocardial stunning for multi-vessel CAD detection. A $p<0.05$ was considered statistically significant. All the statistical analysis was completed using SPSS 13.0 (SPSS Inc., Chicago, IL, USA).

\section{Results}

Among 35 patients with coronary angiography results, 15 patients had multi-vessel CAD, and 20 patients had single-vessel CAD. According to the definition, myocardial stunning was more seen in the multi-vessel CAD rather than single vessel CAD (12/15 vs. $6 / 20, p=0.006)$. And the sensitivity and specificity for multi-vessel CAD detection was $80 \%$ and $70 \%$ respectively. The area under ROC curve was $0.75(p=0.012)$.

Of the 113 patients included in the study, SSS was ranged from 8 to 37 and SDS was 
ranged from 4 to 32 . There were 58 patients had myocardial stunning and 55 patients did not. One example of myocardial stunning is illustrated in Figure 1. Baseline characteristics of the patients are shown in Table 1 and there was no significant difference between the non-stunning and stunning group except SDS, the rate of positive treadmill test, rest EF and TID. There was a moderate correlation between SSS, stress WMS or WTS. Similar moderate correlations between SSS and stress LVMD were observed. A good correlation between stress WMS and WTS was also seen (see Table 2).

In the stunning group, PSD and PHB of contraction were significantly larger during stress than during rest $(15.05 \pm 10.70$ vs. $13.23 \pm 9.01$ and $46.07 \pm 34.29$ vs. $41.02 \pm 32.16, p<0.05)$. PSD and PHB of relaxation were also significantly larger during stress than during rest $(21.21 \pm$ 13.91 vs. $17.46 \pm 10.52$ and $59.03 \pm 37.82$ vs. $52.38 \pm 36.89, p<0.05)$. However, no such changes were observed in the non-stunning group (see Table 3). In the stunning group, EF was significantly lower during stress than during rest $(61.47 \pm 9.74$ vs. $65.67 \pm 9.32, p<0.001)$. EDV and ESV were significantly larger during stress than during rest $(89.22 \pm 25.66$ vs. $84.47 \pm 24.53$ and $36.10 \pm$ 18.28 vs. $30.66 \pm 17.17, p<0.01)$. In the non-stunning group, EDV and ESV showed no significant difference between stress and rest. However, EF was lower during stress than during rest $(59.45 \pm$ 10.13 vs. $61.18 \pm 10.53, p=0.012$ ), which may be caused by the baseline difference of rest $E F$ between two groups $(61.18 \pm 10.53$ vs. $65.67 \pm 9.32, p=0.018)$. As a result, the changes of $E F$ between stress and rest were bigger in the stunning group than in the non-stunning group $(-4.21 \pm$ 4.34 vs. $-1.73 \pm 4.95, p=0.005)$.

For subgroup analysis, there was no difference of LVMD parameters between stress and rest in Group 1, 3 and 4. In Group 2, PSD and PHB of contraction were significantly larger during stress than during rest $(14.08 \pm 5.88$ vs. $11.45 \pm 4.03$ and $42.11 \pm 15.26$ vs. $35.16 \pm 9.85, p<0.01)$. One example is shown in figure 2. PSD and PHB of relaxation were also significantly larger during stress than during rest $(19.65 \pm 9.95$ vs. $15.38 \pm 6.95$ and $54.11 \pm 26.35$ vs. $44.08 \pm 20.68, p<$ 0.01) (see Table 4). 
Among 38 patients in Group 2, 29 patients had myocardial stunning covering only 1 main coronary artery territory (13 in the territory of left anterior descending coronary artery [LAD], 6 in the territory of left circumflex artery $[\mathrm{LCX}]$ and 10 in the territory of right coronary artery $[\mathrm{RCA}])$. Among them, PSD and PHB were significantly larger during stress than during rest in patients with myocardial stunning in the LAD territory rather than in the LCX or RCA territory (15.36 \pm 5.91 vs. $10.04 \pm 3.08$ and $46 \pm 15.64$ vs. $32.15 \pm 9.56$ for contraction, $p<0.01 ; 21.08 \pm 9.63$ vs. $13.38 \pm$ 5.66 and $54 \pm 26.07$ vs. $38.62 \pm 16.15$ for relaxation, $p<0.05$ ) (see Table 5 ).

\section{Discussion}

To our knowledge, this is the first study to directly assess the influence of myocardial stunning to the changes of LVMD parameters between stress and rest gated SPECT MPI. The main finding of our study is that LVMD parameters deteriorate with myocardial stunning especially when there is no myocardial infarction. And for the first time, diastolic LVMD parameters from gated SPECT MPI were analyzed in the occurrence of myocardial stunning. Our results suggested that diastolic LVMD parameters also deteriorated with myocardial stunning. Noteworthy, myocardial stunning in the LAD territory seems to have the most impact on LVMD.

\section{LVMD and myocardial stunning}

The definition of myocardial stunning is various among different studies. Both global and regional LV function parameters have been used as a predictor, such as reduced EF[14], enlarged EDV or ESV[7], and decreased WMS[15-17] or WTS[17]. Sensitivity and specificity are different among these indices for myocardial stunning detection. Santiago et al [17] demonstrated the excellent agreement between WMS and WTS, and suggested that both methods be used, thereby avoiding the potential limitations that were occasionally experienced in post-coronary bypass patients. In this study, we used both WM and WT to analyze the regional LV function (WMSr + WTSr > 1), and had 51\% (58/113) patients with myocardial stunning 60 minutes after exercise test. 
The prevalence of myocardial stunning is also influenced by the different characteristics of patients enrolled in different studies. In the study by Ward et al[15], patients with positive treadmill test results were enrolled. With a mean acquisition time of 54.7 minutes, 12 of 27 (44\%) patients had myocardial stunning. Tanaka et al[18] reported myocardial stunning in $16 \%$ of the total patients, but in $56 \%$ of the patients with mild-to-moderate ischemia, and in $100 \%$ of the patients with severe ischemia. The stress acquisition time in their study was $>30$ minutes after injection. In this study, we deliberately selected moderate to severe ischemia patients (SSS ranged from 8 to 37 and SDS ranged from 4 to 32) for the purpose of myocardial stunning analysis.

As another global LV function parameter, LVMD from SPECT is evaluated by the well-established phase analysis technique, which measures the amplitude (systolic WT) and phase from the regional LV count changes throughout the cardiac cycle as obtained from gated SPECT MPI[1]. It can reliably characterize the changes of LVMD when myocardial stunning is present. As mentioned above, we demonstrated that LVMD deteriorated with myocardial stunning.

\section{LVMD and myocardial infarction}

Previous studies have demonstrated that LVMD correlated with scar burden[19]. In recognition of this, researchers have utilized separate cutoffs for scar and dyssynchrony to identify cardiac resynchronization therapy responders. Ludwig et al[20] found that LVMD characterized by PSD could be spuriously increased by scar. In prior studies, both fixed defect and normal pattern of MPI predicted reduced LVMD parameters during early stress imaging $[3,4,6]$, which was on the opposite of ischemia pattern. We observed the same trend of LVMD in the group of myocardial infarction without stunning. However, when myocardial stunning existed, LVMD parameters tended to increase during stress. In this condition, LVMD was actually affected by both myocardial stunning and myocardial infarction in the opposite direction, but by myocardial stunning the most.

\section{LVMD and coronary artery}


Huang et al[21] firstly demonstrated the relation between early post-stress dyssynchrony and angiographic CAD using TI-201 gated SPECT MPI. In their study, patients with severe multi-vessel CAD had significantly more global and territorial dyssynchrony at stress than at rest. Huang et al[21] further found that LAD stenosis had a higher impact on dyssynchrony than the stenosis in other coronary arteries. In our study, myocardial stunning of the LAD territory also had the most impact on LVMD. The mechanism may be that LAD generally supplies blood to a larger LV territory than other vessels, especially the septal area. In the absence of CAD, patients with left bundle branch block often have diminished myocardial perfusion at the septal area. On the other hand, cardiac resynchronization therapy can restore ventricular septal myocardial perfusion in patients with nonischemic cardiomyopathy presenting with left bundle branch block [22].

\section{Clinical implications}

Comparison of LVMD during stress and rest may provide additional non-perfusion markers of CAD, especially for balanced ischemia of multi-vessel CAD. As reported by Huang et al [21], patients with severe multi-vessel CAD had the worst LVMD at stress than at rest. In our study, LVMD deterioration was more often seen with myocardial stunning, which was related with multi-vessel CAD. Furthermore, when coupled with perfusion defect scores, worsening LVMD may provide incremental prognostic information as reduced EF during stress [23]. In prior studies, the prognostic values of LVMD have already been demonstrated in patients with end-stage renal disease [24], LV dysfunction with CAD [25] and non-ischemic cardiomyopathy [26].

\section{Study limitation}

Firstly, different types of stress tests have been reported to result in myocardial stunning such as exercise, dipyridamole and adenosine. Perfusion abnormalities during dipyridamole or adenosine stress reflect heterogeneity of coronary reserve, which may not be considered as true ischemia. In this study, we only analyzed the changes of LVMD under exercise test. Further studies should be conducted to assess the impact of types of stress on LVMD parameters. 
Secondly, LVMD parameters were slightly increased with myocardial stunning in the infarction group. We did not know if this kind of change would also be seen in heart failure patients with severe low EF, such as 20 - 40\%. Thirdly, LVMD parameters based on gated SPECT MPI varied among software programs [27]. Whether the same results can be demonstrated using other software packages is not clear. Finally, not all patients had coronary angiography, which may lead to systemic bias of statistics. However, the value of myocardial stunning for multi-vessel CAD detection has been reported elsewhere[28].

\section{New Knowledge Gained}

LVMD parameters deteriorate with myocardial stunning especially when there is no myocardial infarction. For the first time, diastolic LVMD parameters from gated SPECT MPI were analyzed in the occurrence of myocardial stunning. Our results suggested that diastolic LVMD also deteriorated with myocardial stunning. Noteworthy, myocardial stunning in the LAD territory seems to have the most impact on LVMD.

\section{Conclusion}

Both systolic and diastolic LVMD parameters deteriorate with myocardial stunning especially when there is no myocardial infarction. This kind of change may have incremental values to diagnose CAD. 


\section{References}

1. Chen J, Garcia EV, Folks RD, Cooke CD, Faber TL, Tauxe EL, et al. Onset of left ventricular mechanical contraction as determined by phase analysis of ECG-gated myocardial perfusion SPECT imaging: development of a diagnostic tool for assessment of cardiac mechanical dyssynchrony. Journal of nuclear cardiology : official publication of the American Society of Nuclear Cardiology 2005; 12 (6):687-695.

2. Aljaroudi W, Koneru J, Heo J, Iskandrian AE. Impact of ischemia on left ventricular dyssynchrony by phase analysis of gated single photon emission computed tomography myocardial perfusion imaging. Journal of nuclear cardiology : official publication of the American Society of Nuclear Cardiology 2011; 18 (1):36-42.

3. Chen CC, Shen TY, Chang MC, Hung GU, Chen WC, Kao CH, et al. Stress-induced myocardial ischemia is associated with early post-stress left ventricular mechanical dyssynchrony as assessed by phase analysis of 201Tl gated SPECT myocardial perfusion imaging. European journal of nuclear medicine and molecular imaging 2012; 39 (12):1904-1909.

4. Singh H, Patel CD, Sharma P, Naik N, Singh S, Narang R. Does perfusion pattern influence stress-induced changes in left ventricular mechanical dyssynchrony on thallium-201-gated SPECT myocardial perfusion imaging? Journal of nuclear cardiology : official publication of the American Society of Nuclear Cardiology 2015; 22 (1):36-43.

5. Zhou Y, Li D, Feng J, Yuan D, Patel Z, Cao K, et al. Left Ventricular Dyssynchrony Parameters Measured by Phase Analysis of Post-stress and Resting Gated SPECT Myocardial Perfusion Imaging. World journal of nuclear medicine 2013; 12 (1):3-7.

6. AlJaroudi W, Alraies MC, DiFilippo F, Brunken RC, Cerqueira MD, Jaber WA. Effect of stress testing on left ventricular mechanical synchrony by phase analysis of gated positron emission tomography in patients with normal myocardial perfusion. European journal of nuclear medicine and molecular imaging 2012; 39 (4):665-672. 7. Hida S, Chikamori T, Tanaka H, Igarashi Y, Shiba C, Usui Y, et al. Diagnostic value of left ventricular dyssynchrony after exercise and at rest in the detection of multivessel coronary artery disease on single-photon emission computed tomography. Circulation journal : official journal of the Japanese Circulation Society 2012; 76 (8):1942-1952.

8. Karacalioglu AO, Balta S, Emer O, Demirkol S, Celik T, Ozguven M. Phase analysis in patients with reversible perfusion defects and normal coronary arteries at angiography. Annals of nuclear medicine 2013; 27 (5):416-422.

9. Peix A, Cabrera LO, Padron K, Rodriguez L, Fernandez J, Lopez G, et al. Association between non-perfusion parameters and presence of ischemia in gated-SPECT 
myocardial perfusion imaging studies. Journal of nuclear cardiology : official publication of the American Society of Nuclear Cardiology 2016.

10. Hale SL, Kloner RA. Acetaminophen and myocardial stunning after transient ischemia in rabbit hearts. Journal of cardiovascular pharmacology and therapeutics 2005; 10 (2):121-129.

11. Li D, Feng J, Yuan D, Cao K, Chen J. Quantification of myocardial perfusion SPECT studies in Chinese population with Western normal databases. J Nucl Cardiol 2010; 17 (3):486-493.

12. Chamuleau SA, Meuwissen M, van Eck-Smit BL, Koch KT, de Jong A, de Winter RJ, et al. Fractional flow reserve, absolute and relative coronary blood flow velocity reserve in relation to the results of technetium-99m sestamibi single-photon emission computed tomography in patients with two-vessel coronary artery disease. Journal of the American College of Cardiology 2001; 37 (5):1316-1322.

13. Forster S, Rieber J, Ubleis C, Weiss M, Bartenstein P, Cumming P, et al. Tc-99m sestamibi single photon emission computed tomography for guiding percutaneous coronary intervention in patients with multivessel disease: a comparison with quantitative coronary angiography and fractional flow reserve. The international journal of cardiovascular imaging 2010; 26 (2):203-213.

14. Mut F, Giubbini R, Vitola J, Lusa L, Sobic-Saranovic D, Peix A, et al. Detection of post-exercise stunning by early gated SPECT myocardial perfusion imaging: results from the IAEA multi-center study. Journal of nuclear cardiology : official publication of the American Society of Nuclear Cardiology 2014; 21 (6):1168-1176.

15. Ward RP, Gundeck EL, Lang RM, Spencer KT, Williams KA. Overestimation of postischemic myocardial stunning on gated SPECT imaging: correlation with echocardiography. Journal of nuclear cardiology : official publication of the American Society of Nuclear Cardiology 2006; 13 (4):514-520.

16. Hacker M, Rieber J, Schmid R, Lafougere C, Tausig A, Theisen K, et al. Comparison of Tc-99m sestamibi SPECT with fractional flow reserve in patients with intermediate coronary artery stenoses. Journal of nuclear cardiology : official publication of the American Society of Nuclear Cardiology 2005; 12 (6):645-654.

17. Santiago JF, Heiba SI, Jana S, Mirzaitehrane M, Dede F, Abdel-Dayem HM. Transient ischemic stunning of the myocardium in stress thallium-201 gated SPET myocardial perfusion imaging: segmental analysis of myocardial perfusion, wall motion and wall thickening changes. European journal of nuclear medicine and molecular imaging 2002; 29 (8):979-983.

18. Tanaka H, Chikamori T, Hida S, Usui Y, Harafuji K, Igarashi Y, et al. Comparison of post-exercise and post-vasodilator stress myocardial stunning as assessed by electrocardiogram-gated single-photon emission computed tomography. Circulation journal : official journal of the Japanese Circulation Society 2005; 69 
(11):1338-1345.

19. Trimble MA, Borges-Neto S, Honeycutt EF, Shaw LK, Pagnanelli R, Chen J, et al. Evaluation of mechanical dyssynchrony and myocardial perfusion using phase analysis of gated SPECT imaging in patients with left ventricular dysfunction. Journal of nuclear cardiology : official publication of the American Society of Nuclear Cardiology 2008; 15 (5):663-670.

20. Ludwig DR, Friehling M, Schelbert EB, Schwartzman D. Impact of scar on SPECT assay of left ventricular contraction dyssynchrony. European journal of nuclear medicine and molecular imaging 2014; 41 (3):529-535.

21. Huang WS, Huang CH, Lee CL, Chen CP, Hung GU, Chen J. Relation of early post-stress left ventricular dyssynchrony and the extent of angiographic coronary artery disease. Journal of nuclear cardiology : official publication of the American Society of Nuclear Cardiology 2014; 21 (6):1048-1056.

22. Ogano M, Iwasaki YK, Tanabe J, Takagi H, Umemoto T, Hayashi M, et al. Cardiac resynchronization therapy restored ventricular septal myocardial perfusion and enhanced ventricular remodeling in patients with nonischemic cardiomyopathy presenting with left bundle branch block. Heart rhythm 2014; 11 (5):836-841.

23. Thomas GS, Miyamoto MI, Morello AP, 3rd, Majmundar H, Thomas JJ, Sampson $\mathrm{CH}$, et al. Technetium $99 \mathrm{~m}$ sestamibi myocardial perfusion imaging predicts clinical outcome in the community outpatient setting. The Nuclear Utility in the Community (NUC) Study. Journal of the American College of Cardiology 2004; 43 (2):213-223.

24. Aggarwal H, AlJaroudi WA, Mehta S, Mannon R, Heo J, Iskandrian AE, et al. The prognostic value of left ventricular mechanical dyssynchrony using gated myocardial perfusion imaging in patients with end-stage renal disease. Journal of nuclear cardiology : official publication of the American Society of Nuclear Cardiology 2014; 21 (4):739-746.

25. Uebleis C, Hellweger S, Laubender RP, Becker A, Sohn HY, Lehner S, et al. Left ventricular dyssynchrony assessed by gated SPECT phase analysis is an independent predictor of death in patients with advanced coronary artery disease and reduced left ventricular function not undergoing cardiac resynchronization therapy. European journal of nuclear medicine and molecular imaging 2012; 39 (10):1561-1569.

26. Zafrir N, Nevzorov R, Bental T, Strasberg B, Gutstein A, Mats I, et al. Prognostic value of left ventricular dyssynchrony by myocardial perfusion-gated SPECT in patients with normal and abnormal left ventricular functions. Journal of nuclear cardiology : official publication of the American Society of Nuclear Cardiology 2014; 21 (3):532-540.

27. Nakajima K, Okuda K, Matsuo S, Kiso K, Kinuya S, Garcia EV. Comparison of phase dyssynchrony analysis using gated myocardial perfusion imaging with four software 
programs: Based on the Japanese Society of Nuclear Medicine working group normal database. Journal of nuclear cardiology : official publication of the American Society of Nuclear Cardiology 2016.

28. Higgins JP, Higgins JA, Williams G. Stress-induced abnormalities in myocardial perfusion imaging that are not related to perfusion but are of diagnostic and prognostic importance. European journal of nuclear medicine and molecular imaging 2007; 34 (4):584-595. 
Table 1 Baseline characteristics

\begin{tabular}{|c|c|c|c|}
\hline & Non-stunning & Stunning & $P$ value \\
\hline & $(n=55)$ & $(n=58)$ & \\
\hline Age(years) & $63.07 \pm 9.32$ & $60.21 \pm 10.91$ & 0.137 \\
\hline Male (\%) & $46(83.6)$ & $42(72.4)$ & 0.153 \\
\hline Hypertension (\%) & $38(69.1)$ & $34(58.6)$ & 0.249 \\
\hline Diabetes (\%) & 17 (30.9) & $10(17.2)$ & 0.090 \\
\hline Smoking (\%) & $27(49.1)$ & 22 (37.9) & 0.234 \\
\hline Percutaneous coronary & $11(20)$ & 15 (25.9) & 0.461 \\
\hline intervention (\%) & & & \\
\hline Coronary artery bypass & $3(5.5)$ & $1(1.7)$ & 0.286 \\
\hline graft surgery $(\%)$ & & & \\
\hline Positive treadmill test (\%) & $25(45.5)$ & $38(65.6)$ & $0.033^{*}$ \\
\hline Summed stress score & $13.84 \pm 5.49$ & $16.07 \pm 7.78$ & 0.082 \\
\hline Summed rest score & $3.49 \pm 5.09$ & $3.59 \pm 3.85$ & 0.911 \\
\hline Summed different score & $9.58 \pm 4.29$ & $12.31 \pm 6.98$ & $0.014^{*}$ \\
\hline Rest ejection fraction & $61.18 \pm 10.53$ & $65.67 \pm 9.32$ & $0.018^{*}$ \\
\hline Rest end-diastolic volume & $84.82 \pm 20.03$ & $84.47 \pm 24.53$ & 0.934 \\
\hline Rest end-systolic volume & $33.93 \pm 14.89$ & $30.66 \pm 17.17$ & 0.283 \\
\hline Transient ischemia dilation & $1.01 \pm 0.13$ & $1.08 \pm 0.13$ & $0.003^{*}$ \\
\hline
\end{tabular}


( ${ }^{*}$, statistically significant) 
Table 2 Spearman correlation coefficients between SSS, SWMS, SWTS and SLVMD

\begin{tabular}{lll}
\hline & Correlation coefficients & P value \\
SSS and SWMS & 0.523 & $0.000^{*}$ \\
SSS and SWTS & 0.53 & $0.000^{*}$ \\
SSS and SCPSD & 0.431 & $0.000^{*}$ \\
SSS and SCPHB & 0.440 & $0.000^{*}$ \\
SSS and SRPSD & 0.356 & $0.000^{*}$ \\
SSS and SRPHB & 0.290 & $0.002^{*}$ \\
SWMS and SWTS & 0.811 & $0.000^{*}$
\end{tabular}

(SSS = summed stress score; SWMS = stress wall motion scores; SWTS = stress wall thickening scores; SLVMD = stress left ventricular mechanical dyssynchrony; SCPSD = stress contraction phase standard deviation; SCPHB = stress contraction phase histogram bandwidth; SRPSD = stress relaxation phase standard deviation; SRPHB $=$ stress relaxation phase histogram bandwidth; *, statistically significant) 
Table 3 Comparison of LVMD parameters between stress and rest

Non-stunning

Stunning

PSD(contraction)

Stress

Rest

$P$ value

PHB(contraction)

Stress

Rest

$P$ value

PSD(relaxation)

Stress

Rest

$P$ value

PHB(relaxation)

Stress

Rest
$12.52 \pm 3.97$

$12.15 \pm 4.65$

0.554

$38.22 \pm 11.06$

$36.62 \pm 13.71$

0.358

$18.87 \pm 8.70$

$18.12 \pm 8.72$

0.461

$54.87 \pm 24.26$

$51.07 \pm 22.36$
$15.05 \pm 10.70$

$13.23 \pm 9.01$

$0.038^{*}$

$46.07 \pm 34.29$

$41.02 \pm 32.16$

$0.026^{*}$

$21.21 \pm 13.91$

$17.46 \pm 10.52$

$0.001^{*}$

$59.03 \pm 37.82$

$52.38 \pm 36.89$ 


$\begin{array}{lll}P \text { value } & 0.166 & 0.017^{*}\end{array}$

$($ LVMD = left ventricular mechanical dyssynchrony; PSD = phase standard deviation; $P H B=$ phase histogram bandwidth; *, statistically significant) 
Table 4. Changes of LVMD parameters in 4 subgroups

\begin{tabular}{|c|c|c|c|c|}
\hline & Group 1 & Group 2 & Group 3 & Group 4 \\
\hline \multicolumn{5}{|c|}{ PSD(contraction) } \\
\hline Stress & $10.76 \pm 3.26$ & $14.08 \pm 5.88$ & $15.61 \pm 13.24$ & $16.88 \pm 10.26$ \\
\hline Rest & $10.89 \pm 4.49$ & $11.45 \pm 4.03$ & $16.70 \pm 13.42$ & $14.28 \pm 5.5$ \\
\hline$P$ value & 0.85 & $0.006^{*}$ & 0.439 & 0.122 \\
\hline \multicolumn{5}{|c|}{ PHB(contraction) } \\
\hline Stress & $33.89 \pm 11.01$ & $42.11 \pm 15.26$ & $48.25 \pm 47.41$ & $51.15 \pm 28.24$ \\
\hline Rest & $33.97 \pm 14.52$ & $35.16 \pm 9.85$ & $49.85 \pm 51.34$ & $43.55 \pm 15.54$ \\
\hline$P$ value & 0.971 & $0.004^{*}$ & 0.549 & 0.098 \\
\hline \multicolumn{5}{|c|}{ PSD(relaxation) } \\
\hline Stress & $16.22 \pm 8.25$ & $19.65 \pm 9.95$ & $21.64 \pm 14.41$ & $26.06 \pm 14.64$ \\
\hline Rest & $15.13 \pm 7.38$ & $15.38 \pm 6.95$ & $22.71 \pm 13.25$ & $22.05 \pm 10.57$ \\
\hline$P$ value & 0.268 & $0.004^{*}$ & 0.546 & 0.054 \\
\hline \multicolumn{5}{|c|}{ PHB(relaxation) } \\
\hline Stress & $48.37 \pm 18.84$ & $54.11 \pm 26.35$ & $62.50 \pm 44.41$ & $72.15 \pm 40.04$ \\
\hline Rest & $45.69 \pm 19.6$ & $44.08 \pm 20.68$ & $64.40 \pm 52.03$ & $64.25 \pm 27.55$ \\
\hline$P$ value & 0.243 & $0.003^{*}$ & 0.732 & 0.177 \\
\hline
\end{tabular}


$(L V M D=$ left ventricular mechanical dyssynchrony; $P S D=$ phase standard deviation; $P H B=$ phase histogram bandwidth; *, statistically significant) 
Table 5 Changes of LVMD parameters in different coronary artery territory

$$
\operatorname{LAD}(n=13) \quad \operatorname{LCX}(n=6) \quad \operatorname{RCA}(n=10)
$$

\section{PSD(contraction)}

\begin{tabular}{|c|c|c|c|}
\hline Stress & $15.36 \pm 5.91$ & $10.35 \pm 1.16$ & $12.39 \pm 3.95$ \\
\hline Rest & $10.04 \pm 3.08$ & $11.52 \pm 2.16$ & $11.21 \pm 2.94$ \\
\hline$P$ value & $0.005^{*}$ & 0.399 & 0.508 \\
\hline \multicolumn{4}{|c|}{ PHB(contraction) } \\
\hline Stress & $46 \pm 15.64$ & $33.5 \pm 4.23$ & $39.5 \pm 10.36$ \\
\hline Rest & $32.15 \pm 9.56$ & $36 \pm 6.72$ & $34.7 \pm 7.64$ \\
\hline$P$ value & $0.002^{*}$ & 0.521 & 0.273 \\
\hline \multicolumn{4}{|c|}{ PSD(relaxation) } \\
\hline Stress & $21.08 \pm 9.63$ & $16.28 \pm 9.6$ & $16.49 \pm 7.39$ \\
\hline Rest & $13.38 \pm 5.66$ & $14.72 \pm 5.34$ & $16.89 \pm 7.64$ \\
\hline$P$ value & $0.007^{*}$ & 0.615 & 0.862 \\
\hline
\end{tabular}

\section{PHB(relaxation)}

$\begin{array}{llll}\text { Stress } & 54 \pm 26.07 & 45.5 \pm 21.46 & 48.4 \pm 18.42 \\ \text { Rest } & 38.62 \pm 16.15 & 45.17 \pm 14.67 & 47.3 \pm 22.16 \\ & & & \\ \text { P value } & 0.011^{*} & 0.946 & 0.796\end{array}$

$($ LVMD = left ventricular mechanical dyssynchrony; $P S D=$ phase standard deviation; $P H B=$ phase histogram bandwidth; * , statistically significant) 


\section{Figure Legends}

Fig.1 An example patient with myocardial ischemia and stunning in the LAD territory. Both the territories of LAD and LCX have myocardial ischemia, but reduced dyskinesis only appears in the LAD territory. LAD: left anterior descending coronary artery; LCX: left circumflex artery; RCA: right coronary artery. 
Fig.2 One example of LVMD changes in patients of Group 2 (myocardial ischemia with stunning). PSD and PHB of contraction were deteriorated during stress than during rest (29.4 vs. 15.5 and 82 vs. 46). LVMD: left ventricular mechanical dyssynchrony; PSD: phase standard deviation; PHB: phase histogram bandwidth. 
Figure 1

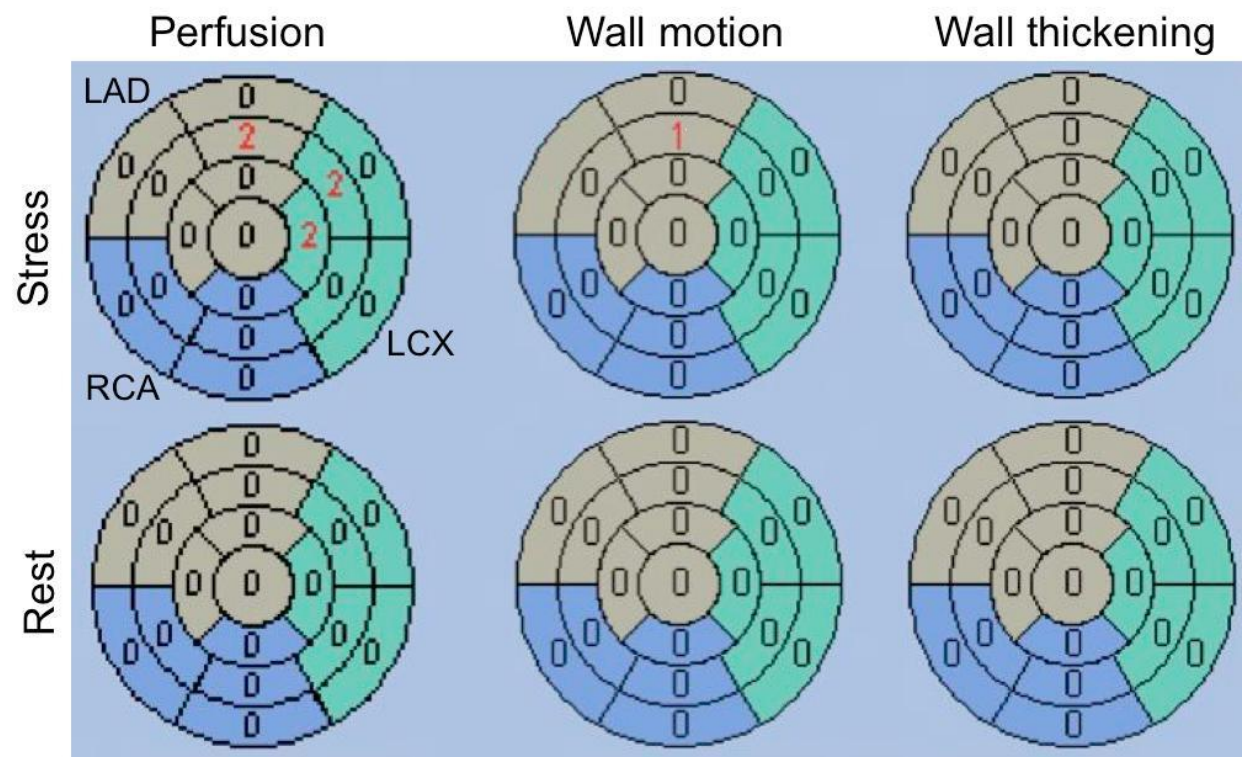


Figure 2

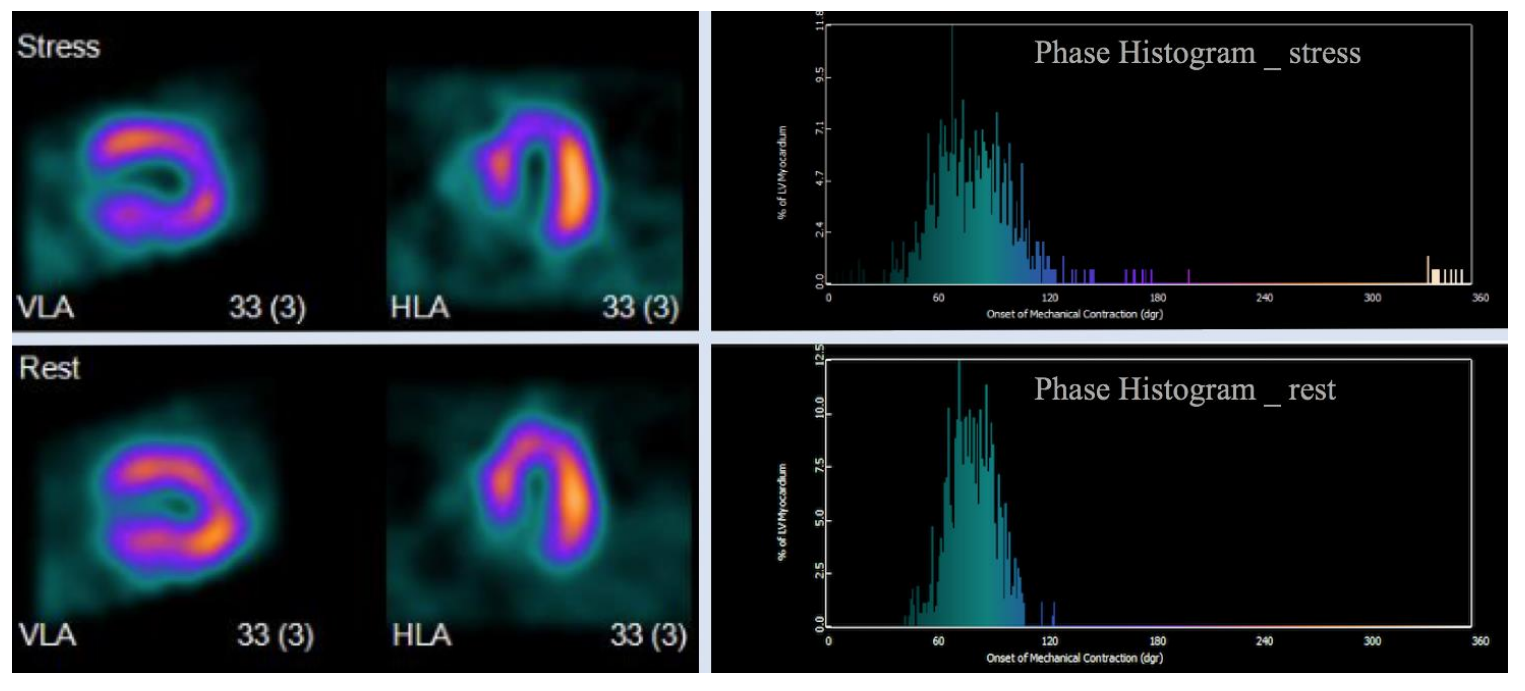

